\title{
Photoswitchable stable charge-distributed states in a new cobalt complex exhibiting photo-induced valence tautomerism $\uparrow$
}

\author{
Michael Slota, ${ }^{* a b}$ Marian Blankenhorn, ${ }^{a}$ Eric Heintze, ${ }^{a}$ Minh Vu, ${ }^{a}$ \\ Ralph Hübner ${ }^{a}$ and Lapo Bogani ${ }^{\star b}$
}

Received 22nd May 2015, Accepted 2nd June 2015

DOI: $10.1039 / c 5 f d 00088 b$

We report the synthesis and magnetic and photomagnetic behaviour of a novel valence tautomeric cobalt complex, [Co(3,5-dbbq) $2_{2}(\mu$-bpym)] (1) (3,5-dbbq $=3,5$-di-tert-butyl1,2 -benzoquinone and $\mu$-bpym $=2,2^{\prime}$-bipyrimidine). The synthesis is performed by reacting $\mathrm{CO}_{2}(\mathrm{CO})_{8}$ and $\mu$-bpym in the presence of the ligand $3,5-\mathrm{dbbq}$ in a mixed solvent under inert atmosphere. The magnetic behavior clearly shows the presence of electron transfer from the catecholate ligand to the cobalt center, producing valence tautomers of [Co" $\left.(\mathrm{SQ})_{2}\right]$ with a transition temperature $\left(T_{1 / 2}\right)$ of $215 \mathrm{~K}$. Photomagnetic studies, performed via both SQUID magnetometry and X-band electron paramagnetic resonance, show the clear presence of photoinduced valence tautomerism, at temperatures considerably higher than previous systems. A metastable charge distribution is observed, strengthening previous investigations on the character of mixed valence ligands. Entropy-driven valence tautomeric interconversion is observed, and drives the transition to the most stable charge distribution. The complex has the ability to coordinate and can be used as a photoswitchable building block, with the photomagnetic characterisation evidencing a metastable state lifetime of the photoinduced valence tautomeric process of ca. $2.9 \times 10^{4} \mathrm{~s}$ below $20 \mathrm{~K}$. The observed yields are higher than ones in similar systems, showing that tiny changes in the molecular structures may have a huge impact.

\section{Introduction}

The effect of magnetism on light has been fascinating scientists for more than 170 years, since the Faraday effect was discovered, ${ }^{\mathbf{1}}$ and since 1900 also the actions of light on the magnetic properties has found more and more attention. ${ }^{2}$ Modern chemistry and new physical investigation methods have allowed clear evidence of

1. Physikalisches Institut, University of Stuttgart, Pfaffenwaldring 57, 70569 Stuttgart, Germany. E-mail: michael.slota@pi1.physik.uni-stuttgart.de; Tel: +4971168564891

${ }^{b}$ Department of Materials, University of Oxford, 16 Parks Road, OX1 3PH, Oxford, UK. E-mail: lapo.bogani@ materials.ox.ac.uk; Tel: +44 (0)1865 283341

$\dagger$ Electronic supplementary information (ESI) available. See DOI: 10.1039/c5fd00088b 
phenomena where the magnetization state can be tuned back and forth by external stimuli, such as light, as well as temperature, pressure, current or chemical doping. ${ }^{3-14}$ For the development of new nanodevices, the individual building blocks need to be reduced down to the molecular level and need to be addressed with high precision. This thus necessitates systems with molecular bistability that can be switched using light. Recent attempts at creating multifunctional and bistable molecular complexes that possess a switchable magnetic functionality have led to novel methods that switch the magnetic properties of nanomagnetic systems, such as single-molecule magnets ${ }^{15,16}$ and single chain magnets. ${ }^{17,18}$ In all these effects some prominent examples of phenomena that trigger the molecular magnetic bistability are complexes which display valence tautomerism (VT), ${ }^{15,19-22}$ spin crossover (SCO) behavior ${ }^{23-25}$ or photoisomerization processes. ${ }^{26}$ VT is a particularly interesting phenomenon, discovered thirty-five years ago by Buchanan and Pierpont, ${ }^{27}$ that is constituted by an intramolecular electron transfer (ET) process. These compounds usually undergo intramolecular ET between the coordinated metal center and the surrounding ligands, while this phenomenon is often accompanied by SCO behaviour of the metal ion, owing to the closely spaced d levels. The coordination compounds of the Co-catecholate/ semiquinone families are known to display VT, and several examples have been reported, attracting considerable interest. ${ }^{7,10,21,22,28-32}$ Usually, the VT mechanism implies electron transfer from the ligands, leading to the interconversion between the low-spin $\mathrm{Co}^{\mathrm{III}}$-catechole (Cat) and high-spin $\mathrm{Co}^{\mathrm{II}}$-semiquinone (SQ) triggered by the presence of redox-active quinone ligands. In these systems, the valence tautomeric process can be schematised as follows:

$$
\left[\mathrm{Co}^{\mathrm{III}}(\mu-\mathrm{bpym})(\mathrm{Cat})(\mathrm{SQ})\right]^{\mathrm{LS}} \rightleftharpoons\left[\mathrm{Co}^{\mathrm{II}}(\mu-\mathrm{bpym})(\mathrm{SQ})_{2}\right]^{\mathrm{HS}}
$$

where LS and HS indicate low-spin and high-spin states, respectively, Cat and SQ indicate the catecholate and semiquinonate oxidation states of the dioxolene, respectively, and $\mu$-bpym is the bipyrimidine ligand that was used in this work. The ET process is entropy-favoured at high temperatures, and shall induce a change of the electronic configuration. The system (Fig. 1) passes from the $S=1 / 2$ ground state (which results from a LS-cobalt ${ }^{\mathrm{III}}\left(S=0, \mathrm{t}_{2 \mathrm{~g}}^{6}\right)$, a Cat $\left(S=0, \pi^{*^{2}}\right)$, and SQ $\left.\left(S=1 / 2, \pi^{* 1}\right)\right)$, to a magnetic molecule formed by an HS-cobalt ${ }^{\mathrm{II}}$ with an unquenched orbital contribution $\left(S=3 / 2, \mathrm{t}_{2 \mathrm{~g}}^{5} \mathrm{e}_{\mathrm{g}}^{2}\right)$ and two SQ ligands $\left(S=1 / 2, \pi^{* 1}\right)$. These individual spin centers can display ferro- or antiferromagnetic coupling.

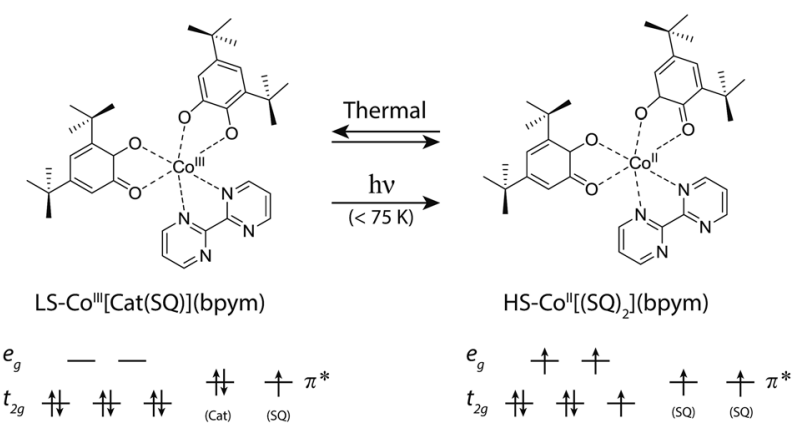

Fig. 1 Schematic diagram of the Co VT complex in the two forms. 
It has been shown that this process can be initiated by light-induced electron transfer, ${ }^{19,33-36}$ which offers an interesting experimental ground for the investigation of photo-induced effects on spin centers. The structural properties are connected to the magnetic bistability providing a fertile ground for the study of molecular and crystal engineering. It is reported that this molecular transition is accompanied with a contraction of the coordination-sphere. ${ }^{21}$ The use of nitrogen-substituted ligands with multi-chelating properties is particularly appealing, because the presence of two binding sites can be used to bind to other metals, and could, in perspective, allow obtaining various phototunable polymetallic compounds, where the Co VT complex is used as a building block in a rational process.

Here we describe the synthesis and photomagnetic and EPR properties of a novel photoswitchable VT complex. We present a compound of the Co-catecholate/semiquinone family that can be used as a phototunable building block for constructing multifunctional valence tautomeric systems. The present system differs from other examples of VT complexes, and in particular from the previously-reported parent system ${ }^{21}$ containing 3,6-di-tert-butyl-1,2-benzoquinone and 2,2'-bipyrimidine by the presence of an exchanged 3,5-di-tert-butyl-1,2-benzoquinone ligand. Hereafter we show that the new system displays enhanced properties, and provide a complete investigation including electron paramagnetic resonance and magnetic measurements under irradiation at different wavelengths and temperatures. By the combined use of these techniques, a metastable state lifetime of the photo-induced valence tautomeric process of $c a .2 .9 \times 10^{4} \mathrm{~s}$ below $20 \mathrm{~K}$ is found, and we determine photoswitching yields much higher than ever presented before, reaching $98 \%$. The results point at the drastic changes that even small variations in the substitution positions in the ligands may induce in such molecular systems.

\section{Materials and methods}

\subsection{Synthesis}

The VT complex $\left[\mathrm{Co}(\mu-\mathrm{bpym})(3,5-\mathrm{dbbq})_{2}\right](\mathbf{1})$ was obtained following procedures similar to those reported in the literature. ${ }^{37}$ All chemicals $\left(\mathrm{Co}_{2}(\mathrm{CO})_{8}, 2,2^{\prime}\right.$-bipyrimidine $(=$ bpym $)$ and 3,5-di-tbutyl-1,2-benzoquinone $(=3,5$-dbbq), were purchased from Sigma-Aldrich and used without further purification except the solvents. The solvents (toluene and hexane) were purified with the standard procedures and degassed via the repeated freeze-pump method ${ }^{38}$ and stored under argon. The synthesis and recrystallization of $\left[\mathrm{Co}(\mu\right.$-bpym $\left.)(3,5-\mathrm{dbbq})_{2}\right]$ was performed under inert gas (Ar) using the standard reversed flow Schlenk technique. Elemental analyses were carried out with a PerkinElmer Analyzer 240.

For the synthesis, $\mathrm{Co}_{2}(\mathrm{CO})_{8}(85.5 \mathrm{mg}, 0.25 \mathrm{mmol})$ and 2,2'-bipyrimidine (87 $\mathrm{mg}, 0.55 \mathrm{mmol}$ ) were stirred for $10 \mathrm{~min}$ in $20 \mathrm{ml}$ toluene. Then 3,5-dbbq ( $220 \mathrm{mg}$, $2 \mathrm{mmol}$ ) in $15 \mathrm{ml}$ toluene was added. The blue mixture was then stirred for an additional $3 \mathrm{~h}$, and then concentrated under reduced pressure and stored overnight at $4{ }^{\circ} \mathrm{C}$. The blue precipitate was then filtered and washed with hexane. The complex was recrystallized from a mixture of toluene $(10 \mathrm{ml})$ and hexane $(30 \mathrm{ml})$. Filtration of the mixture produces a dark-blue solid in $50 \%$ yield. Found: C, $66.20 \%$; $\mathrm{H}, 6.85 \%$; $\mathrm{N}, 8.23 \%$. Calculated for $\mathrm{C}_{36} \mathrm{H}_{46} \mathrm{CoN}_{4} \mathrm{O}_{4}$ : C, 65.74\%; H, 7.05\%; $\mathrm{N}, 8.52 \%$. 


\subsection{Magnetic and photomagnetic measurements}

All magnetic measurements were performed using a commercially available SQUID magnetometer, MPMS-XL7 by QuantumDesign. We prepared the sample in two different ways. First, $2.9 \mathrm{mg}$ of 1 in powder form was filled into the cap of an agar agar capsule (5 mm diameter) of $37 \mathrm{mg}$ mass. Second, a $7.0 \mathrm{mg}$ sample was pressed into a thin pellet of $5 \mathrm{~mm}$ diameter, on which laser power dependent measurements were conducted. To avoid cracking, the pellet was placed between two Suprasil quartz glass platelets of $1 \mathrm{~mm}$ thickness and $5 \mathrm{~mm}$ diameter each. Both samples were fixed in a plastic straw. In order to obtain thinner samples, we tested dissolving the molecule in dichloroethane and then letting the solution dry on top of a glass plate. However, during this process, the sample lost its valence tautomeric property and, accordingly, its photo- and thermally-switchable phase transition (Fig. SI1 $\dagger$ ), underlying the fact that valence tautomeric complexes are quite sensitive to their environment. ${ }^{36,39,40}$

To illuminate the sample and measure its photomagnetic properties on the SQUID magnetometer we developed a home-made system that allows excellent collimation of light onto the sample via an optical fiber. Light from a $532 \mathrm{~nm}$ diode laser ( $15 \mathrm{~mW}$ maximum output power) is guided via a single-mode fiber through a hollow rod into the sample chamber. The output power was controlled via a set of neutral density filters. Prior to each measurement the maximum output power behind the optical fiber end was set to distinct values between $0.2 \mathrm{~mW}$ and $5.4 \mathrm{~mW}$. Owing to the usually large extinction coefficient, only very thin samples were investigated. Later in the paper we will discuss the crucial role of sample preparation for the investigation of light-induced properties.

All magnetic data were obtained at a field of 5000 Oe within $2 \mathrm{~K}$ and $300 \mathrm{~K}$. The sweep rates were set to $0.5 \mathrm{~K} \mathrm{~min}^{-1}$ below $100 \mathrm{~K}$ and $2.5 \mathrm{~K} \mathrm{~min}^{-1}$ above. All data were corrected for the diamagnetic background of the sample and sample holders, as independently determined.

\subsection{EPR and photospectroscopic measurements}

Temperature-dependent X-Band $(9.5 \mathrm{GHz})$ electron paramagnetic resonance (EPR) spectroscopy studies were performed on a Bruker EMXplus system using a universal X-Band resonator ER 4102ST between $300 \mathrm{~K}$ and $3 \mathrm{~K}$ in an Oxford ESR910 cryostat. Approximately $0.2 \mathrm{mg}$ of a powder sample was placed in an open polycarbonate capsule ( $4 \mathrm{~mm}$ diameter) mounted at the bottom of an open quartz glass tube, which ensures good thermal contact. To guide light onto the sample, we fixed an optical fiber with $1 \mathrm{~mm}$ core diameter about $4 \mathrm{~mm}$ above the sample. The calibrated output power of the utilized LOT-QuantumDesign $150 \mathrm{~W}$ Xe arc lamp with a $532 \mathrm{~nm}$ bandpass filter (10 $\mathrm{nm}$ bandwidth) was around $1.5 \mathrm{~mW}$ behind the optical fiber. Via a set of bandpass filters (40 $\mathrm{nm}$ bandwidth each), wavelength dependent absorption between $400 \mathrm{~nm}$ and $850 \mathrm{~nm}$ was investigated.

\section{Results and discussion}

\subsection{Magnetic properties and ESR spectroscopy without irradiation}

The magnetic moment of a pellet sample of $\mathbf{1}$ was recorded during cooling and warming between $2 \mathrm{~K}$ and $300 \mathrm{~K}$ at a field of 5000 Oe (Fig. 2). The pellet form 
ensures that no rearrangement of the sample takes place during the measurement. At $300 \mathrm{~K}$, the $\chi_{\mathrm{m}} T$ value, where $\chi_{\mathrm{m}}$ corresponds to the molar magnetic susceptibility and $T$ is the temperature, is $2.41 \mathrm{emu} \mathrm{K} \mathrm{mol}^{-1}$ and does not reach a saturation point. A $\chi_{\mathrm{m}} T$ around $2.5 \mathrm{emu} \mathrm{K} \mathrm{mol}^{-1}$ is typical for $\mathrm{HS}^{-\mathrm{Co}^{\mathrm{II}}}$ complexes, ${ }^{28}$ which indicates a HS ground state at room temperature. When decreasing the temperature, the sample displays a rapid decrease in $\chi_{\mathrm{m}} T$ centered at $T_{1 / 2}=215 \mathrm{~K}$. This is due to the phase transition from $\mathrm{HS}^{-\mathrm{Co}^{\mathrm{II}}}$ to $\mathrm{LS}^{-\mathrm{Co}^{\mathrm{III}}}{ }^{\text {, where }}$ a charge is transferred from the HS-Co ${ }^{\mathrm{II}}$ ion to the SQ ligand, which is usually accompanied by a contraction of the coordination sphere. ${ }^{21}$ The rapid decrease is followed by a gradual decrease below $200 \mathrm{~K}$ until the system reaches a shouldershaped plateau of $0.97 \mathrm{emu} \mathrm{K} \mathrm{mol}^{-1}$ at $70 \mathrm{~K}$. In contrast to similar Co-based VT compounds, the plateau value does not correspond to the typical spin-1/2 value of $0.38 \mathrm{emu} \mathrm{K} \mathrm{mol}{ }^{-1}$ for $g=2$. This would be expected for a total HS to LS transition, where the paramagnetic signal originates solely from the remaining SQ radicals. The intriguing result could be caused by the presence of a residual amount of a HS-Co ${ }^{\text {II }}$ impurity, which has already been discussed for some Co VT compounds, but at a much lesser extent. ${ }^{28}$ Any other paramagnetic impurities can safely be neglected, as the elemental analysis is coincident with calculations. Moreover, the increased $\chi_{\mathrm{m}} T$ value could also be due to the chosen slow temperature sweep rates, where rearrangements of the charge distributions may occur. ${ }^{28}$ Furthermore, we assume that ferromagnetic exchange mechanisms between the SQ $\pi^{*}$ electrons and the residual $\mathrm{HS}^{-\mathrm{Co}^{\mathrm{II}}}$ ions could also play a role here. ${ }^{29}$ For temperatures below $10 \mathrm{~K}$, the value drops well below $0.9 \mathrm{emu} \mathrm{K} \mathrm{mol}^{-1}$, which we attribute to antiferromagnetic exchange interactions between the spins of the SQ ligands. ${ }^{28}$

When warming up from $2 \mathrm{~K}$ to $300 \mathrm{~K}$, the sample regains its initial susceptibilities. Even after many thermal cycles, the sample does not show any deterioration of the magnetic signal, proving that the switching is fully thermallyreversible.

In order to gain further information on the spin state of the molecule, we conducted EPR spectroscopy in the X-band regime for temperatures between $3 \mathrm{~K}$ and $300 \mathrm{~K}$ (Fig. 3), where no differences between cooling and warming are observed. At $260 \mathrm{~K}$, we observe a small derivative signal centered at 3385(3) G with $g=2.006$ (see Fig. SI $2 \dagger$ ). By cooling down below $220 \mathrm{~K}$, a second derivative signal

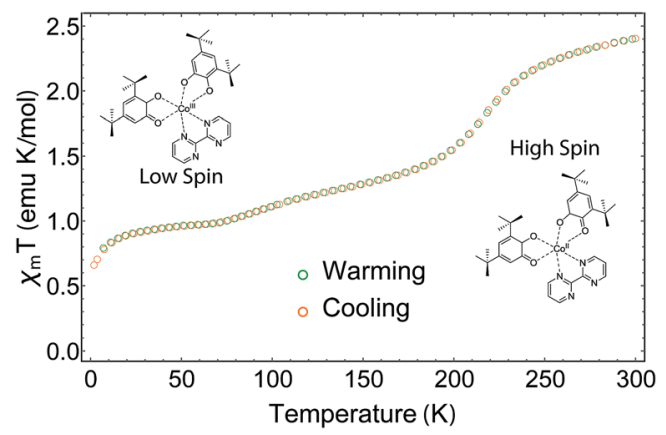

Fig. 2 Temperature dependence of $\chi_{m} T$ of a pellet sample of 1 at an external field of 5000 Oe. 
overlays the spectrum, which is centered at 3376(1) G with $g=2.001$ (see Fig. SI $†$ ), and becomes directly visible below $170 \mathrm{~K}$. Because SQUID measurements give $T_{1 / 2}=215 \mathrm{~K}$ for the HS to LS transition, we assign the transition at $3376 \mathrm{G}$ to the organic radical SQ in the formed LS-Co ${ }^{\mathrm{III}}(\mathrm{Cat})(\mathrm{SQ})$ species. Owing to fast relaxation times, $\mathrm{Co}^{\mathrm{II}}(\mathrm{SQ})_{2}$ complexes are reported to be EPR-silent ${ }^{34}$ and thus not observable at higher temperatures. As both signals show a similar temperature dependence when corrected for the Boltzmann population effect, the second signal may arise from unavoidable impurities or anisotropies of the microcrystalline samples. Therefore it is valid to analyse the EPR data by collecting the signal maxima and minima. Upon further lowering the temperature, the $3376 \mathrm{G}$ signal dominates the spectrum, which therefore gives the proof of an increasing amount of spin-1/2 species. Further we could also observe a small plateau around $70 \mathrm{~K}$, which falls in line with the SQUID plateau. Below $70 \mathrm{~K}$, when the thermal phase-transition is complete, the signal rises exponentially with temperature due to Boltzmann distribution of states.

At low temperatures, we observed a small derivative signal centered at $1700 \mathrm{G}$, which is approximately 500 times smaller than the sharp spin-1/2 signal (Fig. SI $4 \dagger$ ). We attribute this second signal to a $\Delta M= \pm 2$ transition with $g=4$, which arises from the exchange coupling of the radical ligands.

\subsection{The photomagnetic processes}

It has been shown that, after having been cooled down, VT compounds can be switched back into the HS state via irradiation with visible light. Below a certain blocking temperature, the photoexcited metastable state is maintained for very long times, which range from minutes to several days. ${ }^{22}$ Thermal relaxation drives the system back into a ground state, which can be described by an Arrhenius law

$$
\tau=\tau_{0} \exp \left(\Delta / k_{\mathrm{B}} T\right)
$$

( $\tau$ : relaxation time, $\tau_{0}$ : inverse attempt frequency, $\Delta$ : energy barrier, $k_{\mathrm{B}}$ : Boltzmann constant, $T$ : temperature). Furthermore, the system relaxes via quantum tunneling processes, which dominate at sufficiently low temperatures. In order to describe the magnetization dynamics of our system we performed the previous experiments also under irradiation with light.

a)
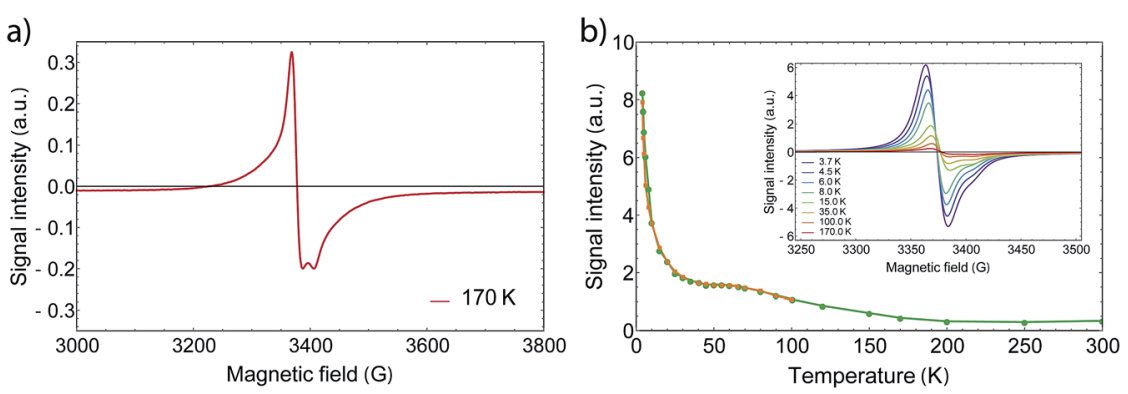

Fig. 3 (a) Derivative EPR signal for 1 at 170 K. (b) Extracted signal intensities versus temperature at fixed microwave power. No thermal hysteresis has been observed between cooling (green) and warming (orange). Inset: X-Band spectrum at different temperatures. 
SQUID magnetometry with light. The low conversion efficiency of photoexcitation experiments is a common issue. Therefore, it is of crucial importance to create a large surface to volume ratio and accordingly thin samples. Solvating the Co VT molecules and drying them on a thin nonmagnetic substrate may not work as these kinds of complexes can deteriorate in solution. ${ }^{29,39}$ Quantitative light power dependent investigations can be carried out on a pellet sample owing to its well-defined shape. However, the high pressure under which the pellet is pressed (1.9 kbar), partially quenched the VT properties (Fig. SI $\dagger$ ). This can be explained by the fact that the high pressure causes a change in the volume of the unit cell, which can be greater than the change caused by thermal contraction or by irradiation. ${ }^{5}$ We achieved the largest conversion efficiency by using a powder sample and pressing it gently in a gelatin capsule. Such samples are less indicated for investigations at high temperatures, as rearrangements of the sample in the externally applied magnetic field may take place, resulting in higher susceptibilities (Fig. SI $1 \dagger$ ). At low temperatures, where Co is in the LS state, no such effects were detected, and we irradiated the powder sample $(2.9 \mathrm{mg})$ with a $532 \mathrm{~nm}$ laser diode at $5.4 \mathrm{~mW}$ for $24 \mathrm{~h}$ at $5 \mathrm{~K}$ and an external field of 5000 Oe. The studies show a distinct effect between the irradiation temperature and a determined critical temperature $T_{\text {LIESST }}$ of $68 \mathrm{~K}$ (Fig. 4 ) defined as the minimum of $\partial \chi_{\mathrm{m}} / \partial T,^{41}$ where the sample goes back into its stable equilibrium state. The determined $T_{\text {LIESST }}$ is about $25 \mathrm{~K}$ higher than the one reported for the 3,6-tertbutyl derivate, ${ }^{21}$ and falls in the range of typical critical temperatures (38-80 K) of other Co VT complexes. ${ }^{42}$ At $5 \mathrm{~K}, \chi_{\mathrm{m}} T$ increases by $20 \%$, from $1.10 \mathrm{emu} \mathrm{K} \mathrm{mol}^{-1}$ to $1.32 \mathrm{emu} \mathrm{K} \mathrm{mol}{ }^{-1}$. The generally higher $\chi_{\mathrm{m}} T$ values, compared to the pellet, are due to rearrangements of the powder sample in the magnetic field. It was not possible to obtain room temperature $\chi_{\mathrm{m}} T$ values, as the main part of excitation takes places close to the surface and a fraction of the light is being reflected by the sample.

Comparable measurements with same laser power on a pressed pellet sample resulted in an approximately four times lower yield. As mentioned before, this could be due to the partially quenched VT induced by pressure or the lower surface to volume ratio compared to powder. Still, pellets offer the possibility to quantitatively investigate the effect of laser power, owing to the defined geometry.
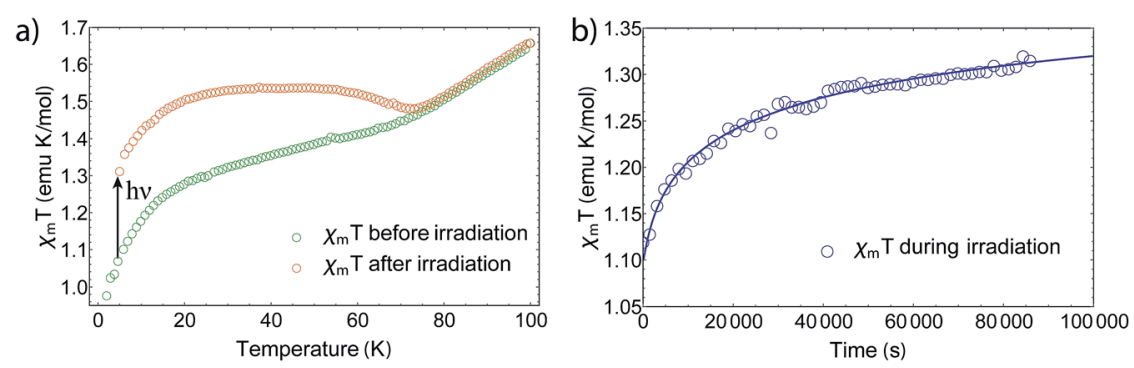

Fig. 4 (a) Change of $\chi_{m} T$ for a powder sample of 1 at an external field of 5000 Oe. After irradiation for $24 \mathrm{~h}$ at $5.4 \mathrm{~mW}, \chi_{\mathrm{m}} T$ increases for about $20 \%$ at $5 \mathrm{~K}$. (b) $\chi_{\mathrm{m}} T$ against time during irradiation and exponential fit ( $\beta=0.33$ and $\tau=26700 \mathrm{~s}$ ). Due to thermal fluctuations of $0.5 \mathrm{~K}$, nonlinear temperature behavior of the susceptibility and very long relaxation time, the fit quality is questionable. 
Due to the Beer-Lambert law and a Gaussian beam profile the excitation fraction scales non-linearly with the laser power (Fig. SI5†). Our investigation shows that increasing the laser power beyond $2 \mathrm{~mW}$ results in increasingly smaller changes of the excitation efficiency. Thus, significantly higher yields can only be achieved via sample preparation in powder form or changing to wavelengths at which the sample shows a lower extinction coefficient (see discussion below).

Furthermore, we also noticed a kink appearing around $12 \mathrm{~K}$ (Fig. 4a) after exciting the sample at $5 \mathrm{~K}$ and increasing $T$. This kink is also reported for the 3,6tert-butyl derivate, ${ }^{21}$ but has not been discussed yet and its origin is still unknown. After heating the sample to room temperature and cooling down to $5 \mathrm{~K}$ again, the complex regains its initial magnetic moment, showing full thermal reversibility with no measurable degeneration. However, we noticed that sample aging (several days) in an external field results in up to $10 \%$ changes of the magnetic moment, which we assume to be caused by different trapping patterns of the differently charged ligands. ${ }^{28}$ By removing the field at room temperature, the magnetization relaxes slowly towards the starting value. The photomagnetic properties are maintained nevertheless.

In order to determine the relaxation behavior, we measured the time evolution of the magnetic moment under irradiation with $532 \mathrm{~nm}$ light at $5 \mathrm{~K}$ (Fig. 4b). Due to photoconversion, the time evolution displays an increasing and saturating behavior, whereby the photostationary limit has not been reached even after $24 \mathrm{~h}$ of irradiation. We fitted the relaxation times via a first-order stretched exponential law given by

$$
a(t)=a_{0} \exp \left(-(t / \tau)^{\beta}\right)
$$

where $a$ represents the $\chi_{\mathrm{m}} T$ time evolution and $\beta$ describes the distribution of relaxation times. The validity of this model has already been approved for spin glasses, ${ }^{43}$ single-molecule magnets ${ }^{44}$ and other Co VT complexes. ${ }^{34,45,46}$ On one hand, a distribution of relaxation times is caused by variations of the internal pressure, since the photo-induced LS to HS transition comes along with a volume change of the molecule itself as indicated by DFT calculations (see ESI $\dagger$ ). On the other hand, as the sample is getting heated up during irradiation of light, thermalisation also plays an important role. After turning off the light the sample needs to assimilate to the ambient temperature and the temperature controller needs to find a new heater equilibrium point.

By fitting we determined the lifetime $\tau$ to be $26700 \pm 5000 \mathrm{~s}$ at $5 \mathrm{~K}$. Thereby, we estimated the heating during irradiation to be $\Delta T=2.45 \mathrm{~K}$, so that the final $\chi_{\mathrm{m}} T$ value matches with the one after irradiation at $5 \mathrm{~K}$. However, it should be noted that the fit does not describe the data well due to thermal instabilities during the measurement and the nonlinear response of $\chi_{\mathrm{m}}$ at low $T \mathrm{~s}$.

The magnetic measurements thus prove that the compound undergoes a longlived transition into a metastable state.

EPR spectroscopy under light irradiation. In order to gain more accurate information on the relaxation dynamics of the excited molecules, we conducted EPR measurements in the X-Band regime under irradiation (see Fig. 5). After 60 min of illumination with $532 \mathrm{~nm}$ light from a Xe arc lamp (1.5 $\mathrm{mW}$ output power, $10 \mathrm{~nm}$ bandwidth) at $3.5 \mathrm{~K}$, the spin-1/2 derivative signal (centered at $3376 \mathrm{G}$ ) decreases by about $75 \%$ (see Fig. SI6 $\dagger$ ). This decrease in signal is due to the photo- 
generated SQ-ligands, which are reported to be EPR-silent as also discussed above. ${ }^{46}$ Thus, the decrease is a measure of the fraction of excitation. When warming up, the signal intensity decreases up to $25 \mathrm{~K}$ and increases again up to 70 $\mathrm{K}$, until it reaches the original signal intensity before irradiation. This is an indication that above $25 \mathrm{~K}$, the relaxation time is becoming comparable with the experimental measurement time. Above $70 \mathrm{~K}$, the signal follows the original pattern with increasing temperature. Compared with the magnetic response on the SQUID magnetometer, we observed a much larger effect of light on the EPR signal which we attribute to the finer powder sample preparation as well as to the selectively higher sensitivity of EPR spectroscopy. Overall the results agree with observations on the SQUID magnetometer.

In order to understand the relaxation mechanisms, we measured the derivative signal intensity at different temperatures for $60 \mathrm{~min}$ after $60 \mathrm{~min}$ of irradiation (Fig. 5b). The relaxation behavior was fitted using two single-exponential laws

$$
A(t)=1-A_{1}(0) \exp \left(-t / \tau_{1}\right)-A_{2}(0) \exp \left(-t / \tau_{2}\right)
$$

where the first one accounts for the molecular relaxation process and the second one for thermal assimilation after the light has been turned off. The extracted longer lifetime $\tau_{1}$ is displayed against the temperature in Fig. 6, whereas the shorter time $\tau_{2}$ that we assume to be caused by thermal assimilation varies independently between $10^{2} \mathrm{~s}$ and $10^{3} \mathrm{~s}$. From the temperature dependence of $\tau_{1}$ we identified a high temperature regime that follows an Arrhenius law eqn (2) with an energy barrier of $\Delta=65.14 \mathrm{~K}$ and $\tau_{1}(0)=1005 \mathrm{~s}$, and a temperatureindependent low temperature regime below $20 \mathrm{~K}$. We note that the fitted energy barrier is close to the critical temperature $T_{\text {LIESST }}$ determined via SQUID. As the relaxation time does not change significantly at low $T$, we assign this relaxation process to pure quantum tunneling. The corresponding tunneling frequency is found to be $3.5 \times 10^{-5} \mathrm{~s}^{-1}$, which is in very good agreement with the results from SQUID magnetometry. This result thus confirms that typical lifetimes fall in the range of $10^{4}-10^{5} \mathrm{~s}$ for Co VT. ${ }^{34}$
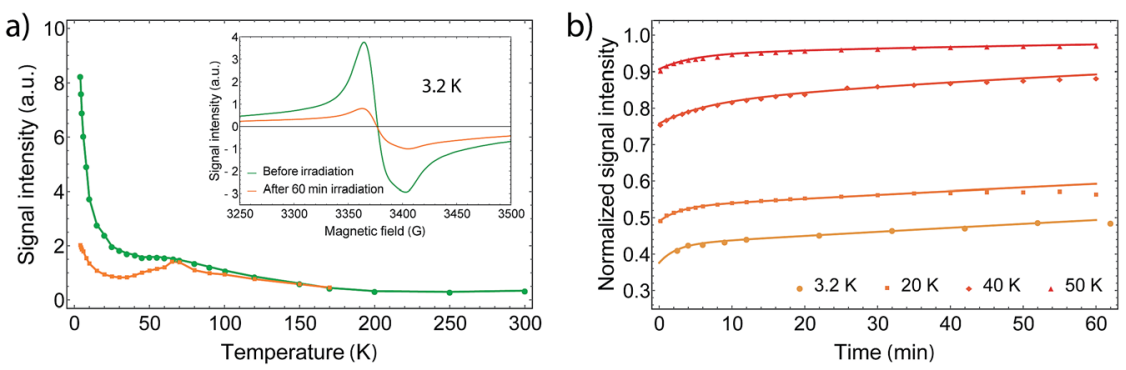

Fig. 5 (a) EPR signal intensity of the derivative signal of the spin-1/2 species while cooling down before (green) irradiation and by warming up after (orange). The inset displays the signal change after 60 min of irradiation (orange); (b) time dependence of the signal relaxation after $60 \mathrm{~min}$ irradiation of $532 \mathrm{~nm}$ light. The data has been fitted using two exponential functions, which account for relaxation and thermal assimilation. 


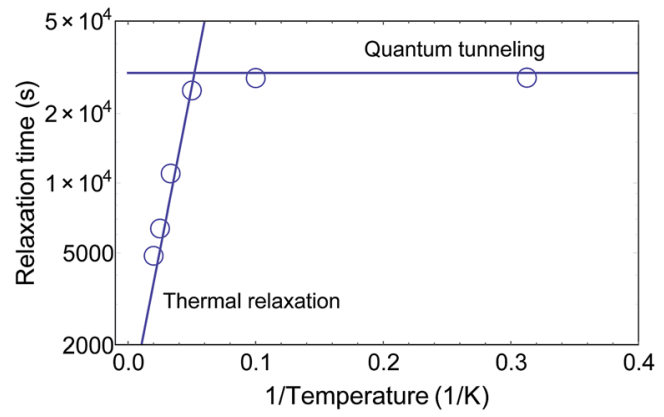

Fig. 6 Temperature dependence of the relaxation time. We identified a high temperature regime following thermal relaxation and a constant low temperature region where quantum tunneling processes dominate.

\subsection{Investigation of possible back-switching}

Eventually, we examined whether it is possible to revert the light-induced switching from LS to HS via illumination of near infrared light at $850 \mathrm{~nm}$. Such behavior has been reported for similar Co VT compounds. ${ }^{42,47}$ While $532 \mathrm{~nm}$ light drives a LMCT process, $850 \mathrm{~nm}$ could induce a HS-LS transition via MLCT. To see a possible back-switching effect, we first irradiated our sample at $3.7 \mathrm{~K}$ in the $\mathrm{X}$ Band spectrometer at $532 \mathrm{~nm}$ for $70 \mathrm{~min}$ and directly exchanged the bandpass filter with a $850 \mathrm{~nm}$ one. If back-switching is present, the X-Band EPR signal

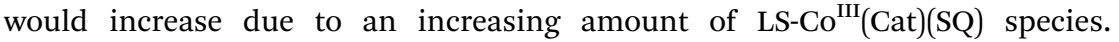
However, the strong opposite is the case and the signal decreased drastically (see Fig. 7), where after $30 \mathrm{~min}$ of irradiation at $850 \mathrm{~nm}$ we even reached a total excitation yield of $98 \%$, and this without the use of a pulsed laser source. Therefore, we can exclude a back-switching behavior in this wavelength region for our sample. The higher conversion efficiency can be related to the lower

a)

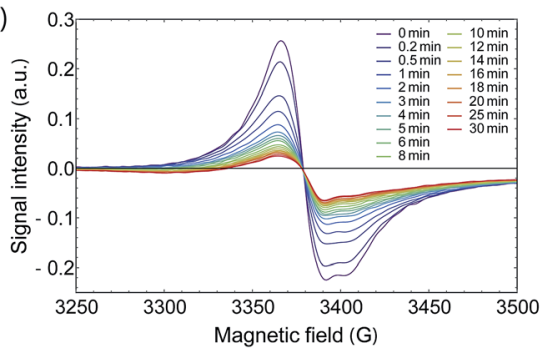

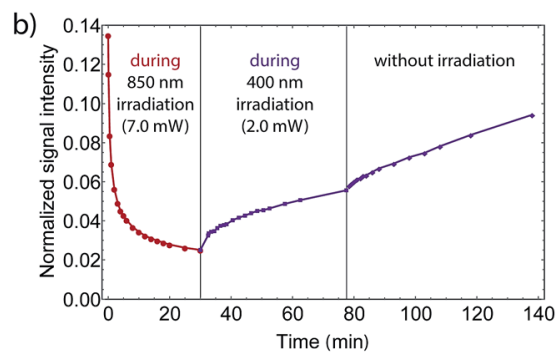

Fig. 7 (a) EPR signal intensity while irradiating with $850 \mathrm{~nm}$ light and $7 \mathrm{~mW}$ light power, after first irradiating for $70 \mathrm{~min}$ at $532 \mathrm{~nm}$ and $1.5 \mathrm{~mW}$. (b) Wavelength dependent performance of the photoabsorption process at $3.75 \mathrm{~K}$. After the first irradiation at $532 \mathrm{~nm}$, the normalized signal was reduced from 1.0 to 0.14 , whereby the signal change is comparable to SI6. $\uparrow$ In contrast to some other Co VT compounds that show back switching behavior, the excitation is enhanced. The signal decreases even further down close to 0.02 , which corresponds to a net excitation of $98 \%$. By changing the wavelength to $400 \mathrm{~nm}$ at $1.5 \mathrm{~mW}$, the signal increases again. As the signal increases faster after turning off the light source, the increase during irradiation at $400 \mathrm{~nm}$ is due to the fact that the relaxation goes faster than the excitation at this wavelength. 
absorption coefficient in the near infrared as determined from UV/Vis/NIR spectroscopy (see Fig. SI7†) and accordingly with a resulting higher penetration depth, so that a higher amount of photons may reach underlying molecules. Also, the output power behind the fiber was strongly enhanced by the four times higher bandwidth, which contributed to the higher yield.

When studying back-switching processes, a larger output power at the deexcitation wavelength is crucial. Otherwise, because the absorption coefficient changes with wavelength, the net excitation fraction may decrease due to counteracting thermal and quantum tunneling relaxation processes. This was exactly the case when we irradiated the sample with $400 \mathrm{~nm}$ subsequently at a lower output power. Moreover, from the UV/Vis/NIR spectrum the absorption at $400 \mathrm{~nm}$ is larger compared to $850 \mathrm{~nm}$ and accordingly the penetration depth is smaller. All in all, we observed an increase of the EPR signal after 45 min of irradiating at $400 \mathrm{~nm}$, which would seem to be a light-induced de-excitation. However, since the signal decreased slightly faster without irradiation, we explain the decrease at 400 $\mathrm{nm}$ to be completely caused by molecular relaxation processes. Finally we did not detect any back-switching from HS to LS between $400 \mathrm{~nm}$ and $850 \mathrm{~nm}$.

\section{Conclusion}

In conclusion we presented the synthesis and magnetic and photomagnetic properties of a novel Co-based VT compound with improved properties. The photomagnetic transition and relaxation process of the complex 1 was thoroughly investigated by SQUID magnetometry and EPR spectroscopy at low temperatures. We were able to identify two relaxation regimes, a temperature independent one due to quantum tunneling below $20 \mathrm{~K}$ and a thermal relaxation that follows an Arrhenius law. Furthermore, we could achieve an almost full excitation at $850 \mathrm{~nm}$ without the use of a pulsed laser. We showed the relevance of sample preparation in order to achieve the best conversion efficiency and demonstrated extremely appealing properties of the compound in terms of temperature and efficiency. We also investigated the power dependence of the effects, showing that laser powers above 2 $\mathrm{mW}$ produce only marginal efficiency increases on the SQUID magnetometer. These results show that the novel complex $\mathbf{1}$ is a superior building block, showing a significantly higher critical temperature $T_{\text {LIESST }}$. Its use in the rational design of novel molecular magnets opens the path to molecular photoswitchable systems and multifunctional materials. Finally, the large efficiency observed now allows pulsed transient EPR measurements to be performed under light irradiation, possibly leading to a clearer understanding of the fundamental processes in VT systems.

\section{Acknowledgements}

We thank the Royal Society via the University Research Fellowship and the University Research Grant, the AvH Stiftung (Sofja Kovalevskaja award) and the European Research Council via the grant ERC-StG-338258-"OptoQMol”.

\section{References}

1 M. Faraday, Philos. Trans. R. Soc. London, 1846, 136, 1-20.

2 J. H. Hart, Am. J. Sci., 1900, 10, 66-73. 
3 C. Cervetti, E. Heintze and L. Bogani, Dalton Trans., 2014, 4220-4232.

4 O. Sato, J. Tao and Y.-Z. Zhang, Angew. Chem., Int. Ed., 2007, 46, 2152-2187.

5 A. Caneschi, A. Dei, F. Fabrizi De Biani, P. Gütlich, V. Ksenofontov, G. Levchenko, A. Hoefer and F. Renz, Chem.-Eur. J., 2001, 7, 3926-3930.

6 F. Matsukura, Y. Tokura and H. Ohno, Nat. Nanotechnol., 2015, 10, 209-220.

7 H. Das, F. Weisser, D. Schweinfurth, C.-Y. Su, L. Bogani, J. Fiedler and B. Sarkar, Chem.-Eur. J., 2010, 16, 2977-2981.

8 D. Schweinfurth, F. Weisser, D. Bubrin, L. Bogani and B. Sarkar, Inorg. Chem., 2011, 50, 6114-6121.

9 A. Droghetti and S. Sanvito, Phys. Rev. Lett., 2011, 107, 047201.

10 P. L. Gentili, L. Bussotti, R. Righini, A. Beni, L. Bogani and A. Dei, Chem. Phys., 2005, 314, 9-17.

11 R. Moroni, R. Buzio, A. Chincarini, U. Valbusa, F. B. de Mongeot, L. Bogani, A. Caneschi, R. Sessoli, L. Cavigli and M. Gurioli, J. Mater. Chem., 2008, 18, 109-115.

12 C. de Julián Fernández, G. Mattei, E. Paz, R. L. Novak, L. Cavigli, L. Bogani, F. J. Palomares, P. Mazzoldi and A. Caneschi, Nanotechnology, 2010, 21, 165701.

13 L. Bogani, L. Cavigli, C. de Julin Fernndez, P. Mazzoldi, G. Mattei, M. Gurioli, M. Dressel and D. Gatteschi, Adv. Mater., 2010, 22, 4054-4058.

14 L. Cavigli, C. de Julin Fernndez, D. Gatteschi, M. Gurioli, C. Sangregorio, G. Mattei, P. Mazzoldi and L. Bogani, J. Magn. Magn. Mater., 2007, 316, e798-e801.

15 O. Sato, Proc. Jpn. Acad., Ser. B, 2012, 88, 213-225.

16 C. Mathonière, H. J. Lin, D. Siretanu, R. Clérac and J. M. Smith, J. Am. Chem. Soc., 2013, 135, 19083-19086.

17 E. Heintze, F. El Hallak, C. Clauß, A. Rettori, M. G. Pini, F. Totti, M. Dressel and L. Bogani, Nat. Mater., 2013, 12, 202-206.

18 T. Liu, H. Zheng, S. Kang, Y. Shiota, S. Hayami, M. Mito, O. Sato, K. Yoshizawa, S. Kanegawa and C. Duan, Nat. Commun., 2013, 4, 1-7.

19 O. Sato, A. Cui, R. Matsuda, J. Tao and S. Hayami, Acc. Chem. Res., 2007, 40, 361-369.

20 D. N. Hendrickson and P. G. Gortlandt, Top. Curr.Chem., 2004, 234, 63-95.

21 J. Dai, S. Kanegawa, Z. Li and S. Kang, Eur. J. Inorg. Chem., 2013, 4150-4153.

22 R. D. Schmidt, D. A. Shultz, J. D. Martin and P. D. Boyle, J. Am. Chem. Soc., 2010, 132, 6261-6273.

23 J.-F. Létard, P. Guionneau and L. Goux-Capes, Top. Curr. Chem., 2004, 235, 221-249.

24 R. Bertoni, M. Cammarata, M. Lorenc, S. F. Matar, J.-F. Létard, H. T. Lemke and E. Collet, Acc. Chem. Res., 2015, 48, 774-781.

25 A. Hauser, Top. Curr. Chem., 2004, 234, 155-198.

26 S. Venkataramani, U. Jana, M. Dommaschk, F. D. Sönnichsen, F. Tuczek and R. Herges, Science, 2011, 331, 445-448.

27 R. M. Buchanan and C. G. Pierpont, J. Am. Chem. Soc., 1980, 102, 4951-4957.

28 M. Affronte, A. Beni, A. Dei and L. Sorace, Dalton Trans., 2007, 5253-5259.

29 A. Witt, F. W. Heinemann, S. Sproules and M. M. Khusniyarov, Chem.-Eur. J., 2014, 20, 11149-11162.

30 X.-Y. Chen, R.-J. Wei, L.-S. Zheng and J. Tao, Inorg. Chem., 2014, 53, 1321213219. 
31 A. Tashiro, S. Kanegawa, O. Sato and Y. Teki, Polyhedron, 2013, 66, 167-170.

32 Y. Teki, M. Shirokoshi, S. Kanegawa and O. Sato, Eur. J. Inorg. Chem., 2011, 2, 3761-3767.

33 O. Sato, J. Photochem. Photobiol., C, 2004, 5, 203-223.

34 A. Beni, A. Dei, D. A. Shultz and L. Sorace, Chem. Phys. Lett., 2006, 428, 400404.

35 C. Carbonera, A. Dei, J. F. Létard, C. Sangregorio and L. Sorace, Angew. Chem., Int. Ed., 2004, 43, 3136-3138.

36 D. M. Adams and D. N. Hendrickson, J. Am. Chem. Soc., 1996, 118, 1151511528.

37 O.-S. Jung, D. H. Jo, Y.-A. Lee, H. K. Chae and Y. S. Sohn, Bull. Chem. Soc. Jpn., 1996, 69, 2211-2214.

38 W. Armarego and C. Chai, Purification of Laboratory Chemicals, ButterworthHeinemann, 2012, p. 1024.

39 Y. Mulyana, G. Poneti, B. Moubaraki, K. S. Murray, B. F. Abrahams, L. Sorace and C. Boskovic, Dalton Trans., 2010, 4757-4767.

40 E. Evangelio, C. Rodriguez-Blanco, Y. Coppel, D. N. Hendrickson, J. P. Sutter, J. Campo and D. Ruiz-Molina, Solid State Sci., 2009, 11, 793-800.

41 J.-F. Létard, L. Capes, G. Chastanet, N. Moliner, S. Létard, J.-A. Real and O. Kahn, Chem. Phys. Lett., 1999, 313, 115-120.

42 T. Tezgerevska, K. G. Alley and C. Boskovic, Coord. Chem. Rev., 2014, 268, $23-$ 40.

43 R. V. Chamberlin, G. Mozurkewich and R. Orbach, Phys. Rev. Lett., 1984, 52, 867-870.

44 C. Sangregorio, T. Ohm, C. Paulsen, R. Sessoli and D. Gatteschi, Phys. Rev. Lett., 1997, 78, 4645-4648.

45 C. Carbonera, A. Dei, C. Sangregorio and J.-F. Létard, Chem. Phys. Lett., 2004, 396, 198-201.

46 C. Carbonera, A. Dei, J.-F. Ltard, C. Sangregorio and L. Sorace, Inorg. Chim. Acta, 2007, 360, 3825-3828.

47 A. Cui, K. Takahashi, A. Fujishima and O. Sato, J. Photochem. Photobiol., A, 2004, 167, 69-73. 